Editorial

\title{
Need of rehabilitation psychology
}

\section{Editorial}

Rehabilitation is the need of those suffering from severe psychiatric illness, motor impairment etc. after a trauma. In order to develop a required skill to carry on their livelihood. In Rehabilitation psychology study one would learn the application of psychological principles on behalf of persons who have disability due to injury or illness. Under the integrated intervention program a rehabilitation psychologists, assess the areas of impairment and empowers a disabled or chronically ill individuals to overcome cognitive, emotional, and functional difficulties, and help to cross the barriers to achieve effective psychological, social and emotional functioning.

Half the battle is won, If you know where to look for, a comprehensive rehabilitation plan does the same for the person with disability. Psychosocial Rehabilitation services are individualized; considering the client's strengths, needs, level of functioning, and preferences. A rehabilitation psychologist serves people across their lifetime since the nature of disability is like that, the typical population groups which comes under this umbrella includes people with traumatic brain injury, stroke, spinal cord injury, limb loss, sensory loss, burn injury, chronic pain, multiple sclerosis, mental retardation, chronic psychiatric illness and neuromuscular disorders. A rehabilitation psychologist not only serves the individual with disability but also to their caregivers/ family members.

In the process of such intervention few points are essential likeRegularity of the treatment, Psycho-education regarding the nature of illness and the prognosis, Promotion of independent living, Prevention of relapse, proper assessment with the help of standardized tests of cognitive and psychological functioning, Coping and adaptation with the treatment. Individual and group interventions has also being found to be effective in such cases in which following types of therapies can be provide like counselling, cognitive remediation, behavioural management, enhancing use of assistive technology, and facilitation of healthy team functioning.

Since the target of a rehabilitation psychologist is to make a
Volume I Issue 2 - 2017

\author{
Pooja Mahour \\ Department of psychiatry, King George's Medical University, \\ India \\ Correspondence: Pooja Mahour, Department of Psychiatry, \\ King George's Medical University, India, \\ Email poojapsy@gmail.com
}

Received: April 27, 2017 | Published: May 08, 2017

dependent individual to independent or near independent one, enhancing patient's quality of life and sense of well- being, which means to facilitating the individual with holistic treatment, hence a rehabilitation psychologist also support or advocates the disabled person in issues related to litigation, government aids, educational institutions, for occupation or employment purpose, public policies etc. for the betterment of disabled, also to attempt to keep the individual in to the main stream of society, in a way they works in collaboration with multimodalities and works in multi-dimension.

A rehabilitation psychologist also involve in to research area in order to provide disable friendly tools, social systems and treatment modalities, they also get involve into teaching and training so that more number of trainers and specialized trainers can be produce. All in all it can be emphasised that rehabilitation psychologist is a need of the time, more number of specialized and trained persons are required thus this skewed area should come in the forefront with appropriate value and reasoning.

\section{Acknowledgements}

None.

\section{Conflict of interest}

The author declares no conflict of interest. 